\title{
Editorial
}

\section{Towards a Europe of health - perspectives for future health policies}

Living and working conditions are powerful determinants of health, physical, mental and social well-being, and productivity. Could and should they be targeted in programs of disease prevention and health promotion, at all levels?

Although health professionals are usually inclined to answer in the affirmative, many political and business decision makers have so far demonstrated little interest in such issues.

\section{Is there a problem?}

Before issuing any "call for action" as to what to promote, and how, they wish to consider whether there is, indeed, a problem -whether stress and depression related problems are widespread, have serious consequences, are becoming more prevalent and severe- and are accessible to interventions.

Available evidence indicates that the answer to all these questions is affirmative. According to the World Health Organization, "mental health problems and stress-related disorders are the biggest overall cause of early death in Europe".

But they are not only a matter of premature mortality. According to the same report, mental ill health and related disorders are among the major health concerns in Europe today. In particular, depression, suicide and other stress-related conditions, together with destructive life-styles and psychosomatic diseases, cause immense suffering to people and their families, as well as placing "a great economic cost on society".

\section{The root causes}

But do we know the root causes of such outcomes, and, if so, are they amenable to change? Mental health problems can be caused by a combination of circumstances: biological, social and psychological factors, and stressful life events. They are usually associated with difficulties either in our personal lives or due to the wider environment in which we live.

In its analysis of such circumstances, the British Government (1998) drew attention to five different types of health determinants, and highlighted each of them. The "fixed" factors (e.g., genes, sex, ageing) are difficult to adjust, whereas successful interventions are feasible against those listed under the other four headings: social and economic (e.g., employment, 
poverty, social exclusion); environmental (e.g., air and water quality, housing, social environment); lifestyle (e.g., diet, physical activity, tobacco, alcohol, drugs); and access to and quality of services (e.g., education, NHS, social services).

The causal significance of the latter four types of factors has been analysed by Wilkinson and Marmot. The authors conclude that the "solid facts" are:

- Social and economic circumstances affect people's health strongly throughout life.

- Work-related stress increases the risk of disease as do unemployment and job insecurity.

- Social exclusion creates health risks, while social support promotes health and well-being.

- Individuals may turn to alcohol, drugs and tobacco and suffer as a result of their use, but this process is also influenced by the wider social setting, which is often beyond individual control.

Sustained work-related stress is an important determinant of depressive disorders. Such disorders are the fourth leading cause of the global disease burden. They are expected to rank second by 2020, behind ischaemic heart disease, but ahead of all other diseases.

It is further likely that sustained work-related stress is an important determinant of the metabolic syndrome. This syndrome comprises a combination of: abdominal accumulation of adipose tissue; a decrease in cellular sensitivity to insulin; dyslipidemia (increase in LDL cholesterol and triglycerides, decrease in HDL cholesterol); and hypertension, probably contributing both to ischaemic heart disease and Diabetes Type 2 morbidity.

\section{The European commission's Guidance on work related stress}

This EU Guidance emphasizes that, according to the EU Framework Directive, employers have a "duty to ensure the safety and health of workers in every aspect related to the work". The Directive's principles of prevention include "avoiding risks", "combating the risks at source", and "adapting the work to the individual". In addition, the Directive indicates the employers' duty to develop "a coherent overall prevention policy". The Commission's Guidance provides a solid basis for such endeavours.

Based on surveillance at individual workplaces and monitoring at national and regional levels, work-related stress should be prevented or counteracted by job redesign (e.g. by empowering the employees, and avoiding both over- and underload), by improving social support and by providing reasonable reward for the effort invested by workers, as integral parts of the overall management system, also for small and medium sized enterprises. And, of course, by adjusting occupational physical settings to the workers' abilities, needs and reasonable expectations Supporting actions should include not only research but also adjustments of curricula in business schools, in schools of technology, medicine and behavioural and social sciences, and in the training and retraining of labour inspectors, occupational health officers, managers and supervisors, in line with such goals.

In the occupational health field, the European Framework Directive, the EU Guidance on work-related stress, a series of activities related to the concept "Corporate Social Responsi- 
bility (CSR)" and the European Framework agreement on work-related stress, covering several hundred million employees in 27 European Union member states, jointly provide a solid basis for integrated health promotion and disease prevention.

The great challenge now is three-fold: (1) to implement available evidence, (2) to evaluate the implementation, and (3) to serve as a model in this field for other countries and continents, to consider, adjust, and assimilate.

Given the triple global burden of high morbidity, low productivity, and wide-spread poverty, there is now an urgent need for such preventive and promotive actions across societal sectors and levels for beneficial outcomes for all concerned - workers, management, and society.

\section{Acknowledgement}

This paper is based on the author's Barchas Award Lecture, American Psychosomatic Society, Budapest, 10 March, 2007.

Lennart Levi, MD, Phd

Emeritus Professor of Psychosocial Medicine

Karolinska Institutet

SWEDEN 